\title{
A Novel Theory for the Scattering of P-Polarized Hermite-Gaussian Electromagnetic Beams by a Double Metallic Nano-Slit
}

\author{
I. Guzman-Montaño ${ }^{1}$, G. Montiel-Gonzalez ${ }^{1}$, J. Sumaya-Martinez ${ }^{1}$, P. Rosendo-Francisco ${ }^{1}$, \& J. Reyes-Gomez ${ }^{2}$ \\ ${ }^{1}$ Facultad de Ciencias, Universidad Autónoma del Estado de México. Instituto Literario \#100 Col. Centro. \\ Toluca, México. C.P. 50000. Correo \\ ${ }^{2}$ Facultad de Ciencias de la Universidad de Colima, Bernal Diaz del Castillo \#340, Col. Villas San Sebastian, \\ Colima, Colima, Mexico. C.P. 28045 \\ Correspondence: J. Sumaya-Martinez, Facultad de Ciencias, Universidad Autónoma del Estado de México. \\ Instituto Literario \#100 Col. Centro. Toluca, México. C.P. 50000. Correo. E-mail: jsm@uaemex.mx
}

Received: October 29, 2018

Accepted: November 30, 2018

Online Published: July 15, 2019

doi:10.5539/apr.v11n4p1

URL: http://dx.doi.org/10.5539/apr.v11n4p1

\begin{abstract}
We present a rigorous theory for oblique incident Hermite-Gaussian beams, diffracted by two optical nano-slits of width $\ell$ and separation d, in a thick metallic screen for the case of polarization TM (P). The far field spectra as a function of several opto-geometrical parameters, wavelength $\lambda$, slit width $\ell$, separation $\mathrm{d}$, incidence angle $\theta_{\mathrm{i}}$ and Hermite order $\mathrm{m}$ is analyzed. In the vectorial diffraction region given when $\lambda />0.2$, where $\ell$ is the incident wavelength and as a function of the separation between slits $d$; we have numerically analyzed: the far field spectra, the energy diffracted along the incident beam direction $\left(E_{i}\right)$, and the validity of an approximate diffraction (scalar) property, namely $E_{i}=N \tau / \lambda$.
\end{abstract}

Keywords: Difracction, Scattering, Nano-Slits

\section{Introduction}

Currently there are several rigorous theories of diffraction by plane electromagnetic waves (Enriquez et al., 2011) and Gaussian beams (Mata et al., 1993, 1994) by two slits in metallic screens of zero thickness. However, these theories do not consider Hermite-Gauss beams or oblique incidence, nor thick screens with nonzero thickness (Mata et al., 2008).

In this paper we present a novel rigorous theory of diffraction that allows to consider the illumination by Hermite-Gaussian beams at oblique incidence onto two nano-slits of width $\ell$ and separation $d$ in screens with infinite conductivity and thickness $h$.

In particular, we analyze the coupling between slits through the numerical study of the diffracted energy along the direction of the incident $\left(E_{i}\right)$ beam energy as a function of the parameter of separation $d$ between the slits. It is revealed the existence of oscillations in the energy $E_{i}$. We also show that in the case of $\operatorname{TM}(\mathrm{S})$ polarization, the energy $E_{i}$ is special because when compared to other diffraction patterns. Finally, we show that the scalar property valid at the scalar region $(\lambda / \ell<0.2) E_{i}=N \tau / \lambda$ (Alvarez-Cabanillas, 1995) is not longer valid.

\section{A Novel Vector Theory of Diffraction}

In Figure 1 we have two slits on a screen of infinite conductivity, and non-zero thickness denoted by $h$. In this screen you have two parallel to the $\mathrm{Oz}$ axis, $\ell$ wide and spaced slits $d$. The display is in the gap and impinges perpendicularly on it a Hermite-Gaussian beam with wavelength $\lambda=2 \pi / k$ and order $\mathrm{m}$. We will use the complex representation for the fields and omit the time factor going forward $e^{-i \omega t} . H$ is the magnetic field when you have the TM (magnetic field parallel to the axis $\mathrm{Oz}$ ) polarization. The $H$ field satisfies the Helmholtz equation (Mata et al., 1994)

$$
\partial^{2} \mathrm{H} / \partial \mathrm{x}^{2}+\partial^{2} \mathrm{H} / \partial \mathrm{y}^{2}+\mathrm{k}^{2} \mathrm{H}=0
$$

Denote by $\mathrm{H}_{\mathrm{I}}$ the solution of Eq (1) in the region I $(y>h / 2)$, expressed by a plane wave expansion:

$$
H_{I}(x, y)=\frac{1}{\sqrt{2 \pi}} \int_{-k}^{k} A(\alpha) e^{i(\alpha x-\beta y)} d \alpha+\frac{1}{\sqrt{2 \pi}} \int_{-k}^{k} B(\alpha) e^{i(\alpha x+\beta y)} d \alpha
$$


The first integral is identified with the incident beam due to the sign of the $\alpha$ and $\beta$ k-components.

In region II, within the screen, $-h / 2<y<h / 2$ the electromagnetic field will be represented by the following modal series:

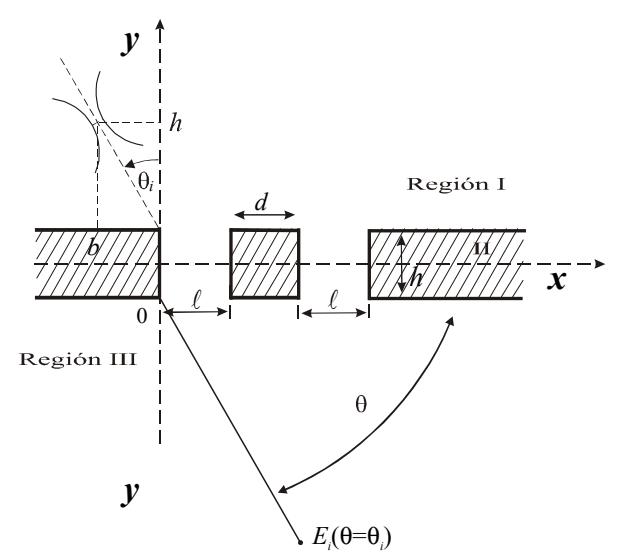

Figure 1. Our system. Two slits of width $\ell$ and spacing $d$ in an infinitely thick conducting screen $h$. The energy diffracted along the incident direction $\left(E_{i}\right)$ is diffracted in the direction of(relative to the axis Oy) $=\theta_{i}$ (From the axis Ox)

$$
\mathrm{H}_{\mathrm{II}}(\mathrm{x}, \mathrm{y})=\sum_{\mathrm{n}=0}^{\infty} \mathrm{a}_{\mathrm{n}}^{1} \varphi_{\mathrm{n}}^{1}(\mathrm{x}, \mathrm{y})+\sum_{\mathrm{n}=0}^{\infty} \mathrm{a}_{\mathrm{n}}^{2} \varphi_{\mathrm{n}}^{2}(\mathrm{x}, \mathrm{y})
$$

Where in $i=1,2$ the set $\varphi_{n}^{i}(x)$, are functions whose normal derivative is zero at the walls for the TM polarization.

The diffracted field below the screen, for $y<-h / 2$ (region III), could be expressed as a plane wave expansion too:

$$
H_{I I I}(x, y)=\frac{1}{\sqrt{2 \pi}} \int_{-\infty}^{\infty} C(\alpha) e^{i(\alpha x+\beta y)} d \alpha
$$

Our goal is to determine the transmitted field (Eq. (4)), for which one needs to determine $C(a)$. Note that $C$ (a) depends on the coefficients $a_{n}^{1}$ and $a_{n}^{2}$ and the incident amplitude $A(a)$. For this, we use the appropriate conditions of continuity, which could be obtained from Maxwell's equations (Alvarez-Cabanillas, 1995). These conditions lead us to the following matrix system, in which the matrix columns $\boldsymbol{a}_{1}$ and $\boldsymbol{a}_{2}$ are formed respectively by the coefficient $a_{n}^{1}$ and $a_{n}^{2}$.

$$
\left\{\begin{array}{l}
M_{11} a_{1}+M_{12} a_{2}=S_{1} \\
M_{21} a_{1}+M_{22} a_{2}=S_{2}
\end{array}\right.
$$

where $M_{i k}(i, k=1,2)$ are square matrices dependent on the opto-geometrical parameters and $S_{i}(i=$ $1,2)$ are matrices depending only on $A(a)$. The determination of the modal coefficients $a_{n}^{1}$ and $a_{n}^{2}$. allow us to calculate the diffracted field in any region for TM polarization.

\section{Results and Discussion}

Using the complex Poynting vector is possible to obtain the diffracted intensity at the angle $\theta_{\mathrm{i}}$. For a Hermite-Gaussian beam, the spectral amplitude is (Mata et al., 2008):

$$
\mathrm{A}(\alpha)=\frac{\mathrm{L}}{2} \mathrm{i}^{\mathrm{m}} \mathrm{H}_{\mathrm{m}}\left[-\frac{\mathrm{L}}{2}\left(\alpha \sin \theta_{\mathrm{i}}-\beta \cos \theta_{\mathrm{i}}\right)\right] \times\left[\sin \theta_{\mathrm{i}}+\left(\frac{\alpha}{\beta}\right) \cos \theta_{\mathrm{i}}\right] \mathrm{e}^{(-\mathrm{i} \alpha \mathrm{b})} \times \mathrm{e}^{\left[-\left(\alpha \sin \theta_{\mathrm{i}}-\beta \cos \theta_{\mathrm{i}}\right)^{2} \mathrm{~L}^{2} / 8\right]}
$$

where $H_{m}$ is the Hermite polynomial of order $\mathrm{m}$. The position of the beam waist is given by the parameter $\mathrm{b}$ (see Figure 1).

In the figures relating to energy diffracted along the direction of the incident beam is $E_{i}\left(\theta=\theta_{i}\right)$ the diffracted angle in the direction of the incident beam, measured from the axis Ox and $\theta_{i}$ is the angle of incident beams to the axis Oy measured. The energy, the diffracted intensity $I(\theta)$ and the transmission coefficient $\tau$ are normalized to the total incident energy. All parameters normalized opto-geometrical width of the slots $\ell$, that is, , $\ell=1$. 
In Figures 2 and 3 show the diffraction patterns of Hermite-Gaussian beams for the fundamental mode $\mathrm{m}=0$ at normal incidence and oblique incidence of $30^{\circ}$; the wavelength of the incident beams is $\lambda \ell \ell=0.9$, with extremely wide Gaussian beams $\mathrm{L} / \ell=500 / \sqrt{2}$ and fixed at the position $b / \ell=0.5$, the thickness of the screen is $h / \ell=1$ and the gaps between slits are $d / \ell=0,1,3.5$ and 5 .

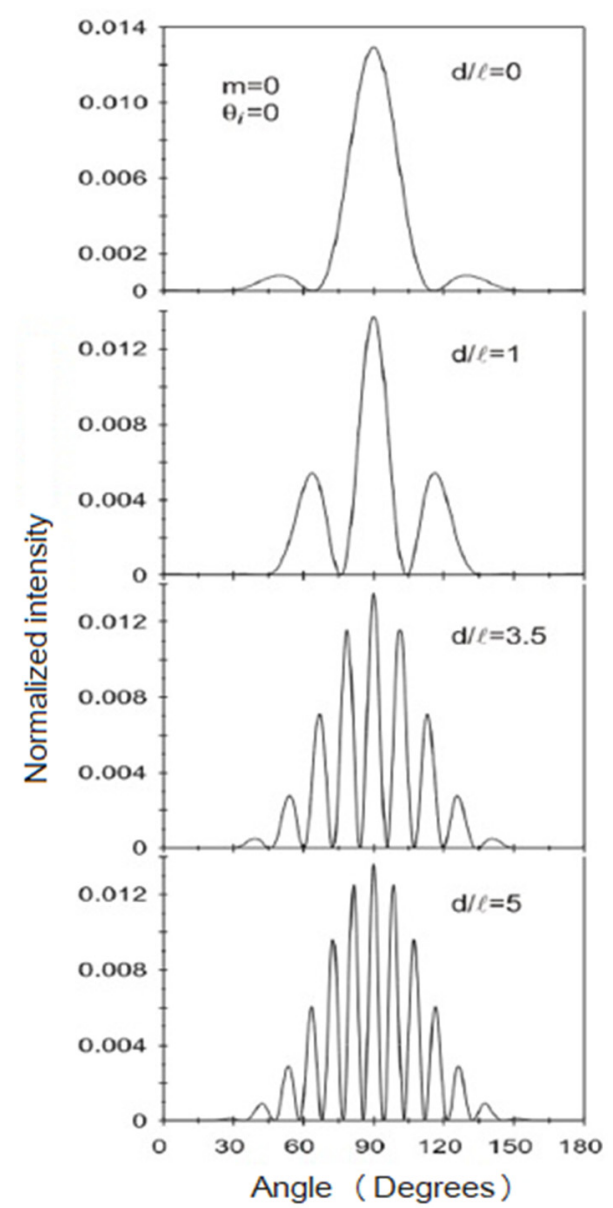

Figure 2. Diffraction patterns normalized $\left(I(\theta) / I_{0}\right)$ of Hermite-Gaussian beams of $\mathrm{m}=0$ normally incident on two slits so. With $\lambda / \ell=0.9, \mathrm{~L} / \ell=500 / \sqrt{2}, h / \ell=1$ and position $b / \ell=0.5$ and for separations $d / \ell=0,1,3.5$ and 5

The shape of the diffraction patterns for the $\mathrm{m}=2$ mode, not shown, is identical to the spectra of FIGURES 2 and 3 (with the same opto-geometrical parameters) except for a scaling factor which provides a lower intensity for this mode, from the respective Hermite polynomial.

From these diffraction patterns we have taken the diffracted energy $E_{i}$ along the direction of the incident beams. Figures 4 and 5 show the behavior of the $E_{i}$ separation according to $d$ for $\mathrm{m}=0$ and 2 modes; opto-geometrical parameters of these figures are the same in Figures 2 and 3.

The curves of Figures 4 and 5 show the oscillatory behaviors as $E_{i}$ a function of the spacing $d$, in particular for the period is normal incidence to oblique incidence $\lambda$ and the period is $2 \lambda$. 


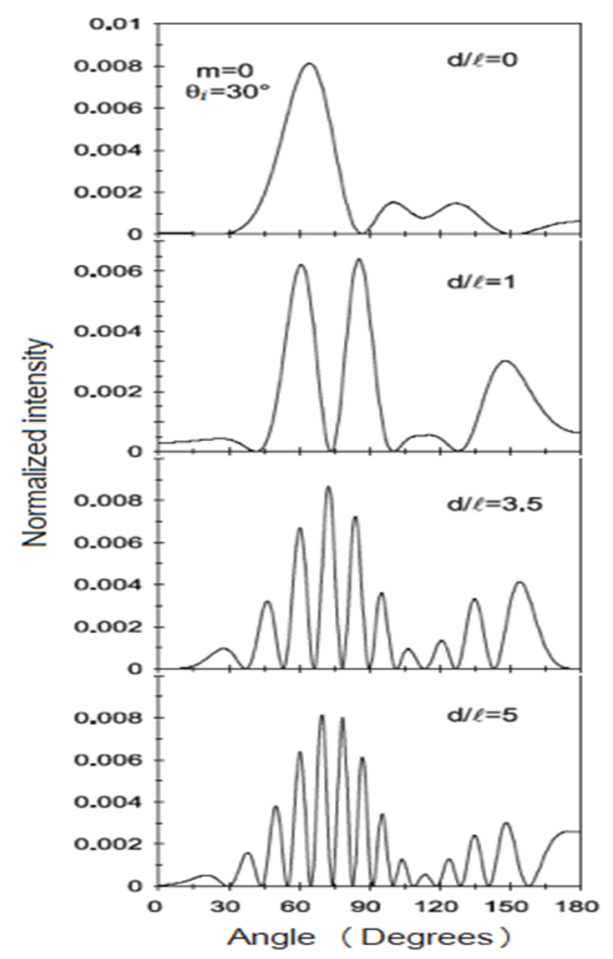

Figure 3. Normalized diffraction patterns $\left(I(\theta) / I_{0}\right)$ Hermite-Gaussian beam for $\mathrm{m}=0$ to $30^{\circ}$ obliquely incident on two slits so. Same parameters of Figure 2

In Figure 5 has also been drawn in broken lines the $2 \tau / \lambda$ function. As you can see, this function does not overlap with the energy with $E_{i}$ which we can say that the property of diffraction $E_{i}=2 \tau / \lambda$ is not valid in the vector region at least for the separation parameter $d$ and doing extremely wide.

Finally, in Figure 6 different diffracted energy around the energy is $E_{i}$. The upper curves of Figure 6 correspond to normal incidence for the $m=2$ mode, with the same parameters of Figure 3; diffracted energies correspond to the angles diffracted $\theta=90^{\circ}, 91^{\circ}, 92^{\circ}$ and $94^{\circ}$. The curves in the lower window of Figure 6 correspond to oblique incidence of $30^{\circ}$, also for mode $m=2$, with the same parameters of Figure 4 . The diffracted energies shown, corresponding to angles diffracted around of $\theta=60^{\circ}$ (corresponding to the diffracted energy along the oblique incidence angle $\theta_{i}=30^{\circ}$ ) and for the angles $58^{\circ}, 57^{\circ}$ and $64^{\circ}$.

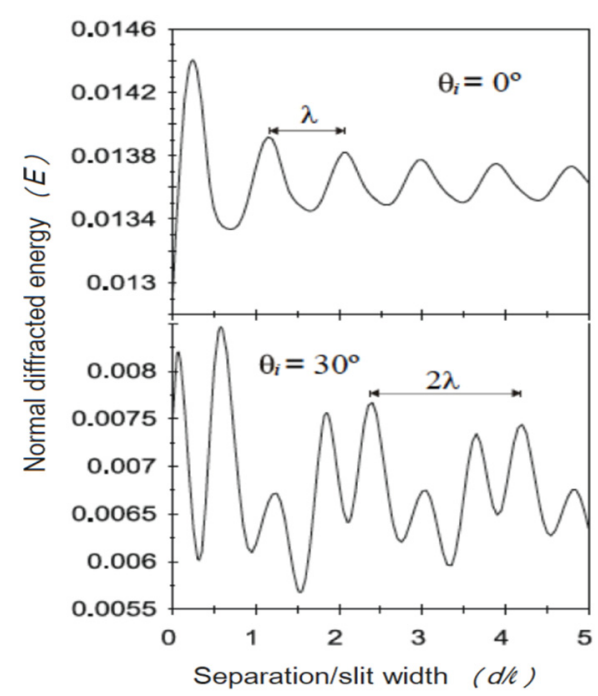

Figure 4. Energy diffracted in the direction normal to the standard $E_{i}$ to Hermite-Gauss beam, depending on the spacing $d / \ell$ screen. For the fundamental mode $m=0$, at normal incidence and oblique incidence of $30^{\circ}$, with $\lambda / \ell=0.9, \mathrm{~L} / \ell=500 / \sqrt{2}, h / \ell=1, \mathrm{y} \mathrm{b} / \ell=0.5$ 


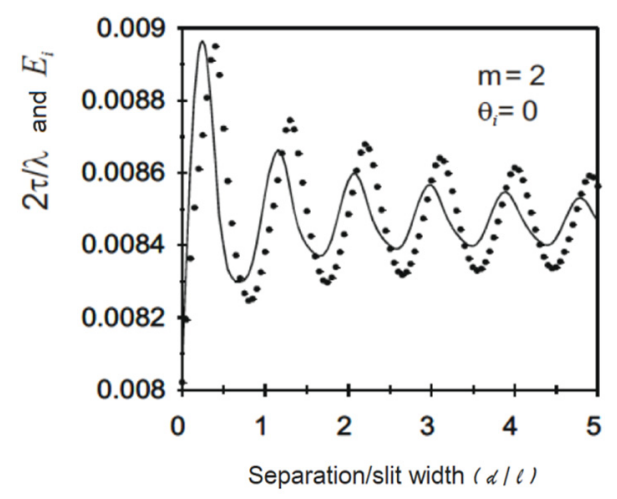

Figure 5. Energy diffracted in the direction normal to the $E_{i}$ (solid line) Hermite-Gauss beam, thus $m=2$ and $2 \tau / \lambda$ property (dashed line), in function of the spacing $d / \ell$. Same parameters of Figure 3

Energy analyzing energy diffracted $E_{i}$ around for $m=0$ at normal incidence and oblique incidence of $30^{\circ}$ as also carried out (data not shown) found similar patterns for mode $m=2$ (see Figure 6), the energy diffracted around the energy as $E_{i}$ a function of the spacing $d$, decay to zero.

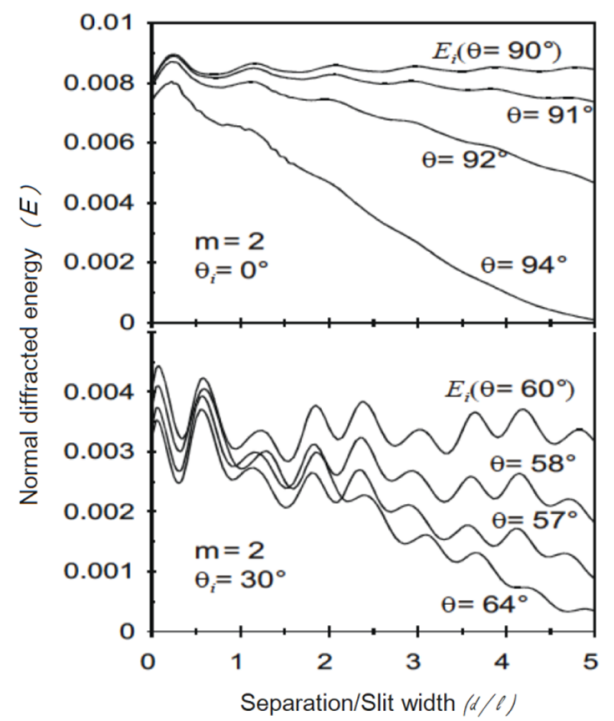

Figure 6. Energy diffracted around energy $E_{i}(\theta=\theta i)$ Hermite-Gauss beam, for the $m=2$ mode according to the distance between slits $d / \ell$. Same parameters of Figure 4

\section{Conclusions}

We present a rigorous theory of diffraction for the oblique incidence beam Hermite-Gaussian (HG) on a screen of thickness $h$ with wide slits separating slits $\ell$ and $d$. In the case of TM(S) polarization and wavelengths in the vector region $\frac{\lambda}{\ell}>0.2$, we have found that the diffracted along the direction of the incident beam energy has oscillations period $\lambda$ as a function of the spacing $\mathrm{d}$ for modes $m=0$ and 2 , and $\lambda$ for the period $2 \lambda$ at $30^{\circ}$ oblique incidence. Finally, we note that the energy has special characteristics compared diffracted energies in other directions and found numerically that ownership of scalar diffraction $(\lambda / \ell<0.2)$ given by $E_{i}=2 \tau / \lambda$ is no longer valid in this region $(\lambda / \ell>0.2)$.

\section{Conflict of interests}

The authors declare that there is no conflict of interests regarding the publication of this paper. 


\section{References}

Enriquez-Leon, G., Martinez-Flores, M. G., Torres-Morales, G., \& Sumaya-Martinez, J. (2011). Rigorous diffraction of Hermite-Gaussian beams by a double slit. TM polarization. Eight Symposium Optics in Industry.

Mata-Méndez, O., \& Chávez-Rivas, F. (1993). Estudio teórico y numérico de la difracción en óptica electromagnética. II Teoría rigurosa de la difracción de un haz gaussiano por dos rendijas. Polarización TE. Revista Mexicana de Física, 39(5), 706-721.

Mata-Méndez, O., \& Álvarez-Cabanillas, M. A. (1994). Estudio teórico y numérico de la difracción en óptica electromagnética. III. Teoría rigurosa de la difracción de un haz gaussiano por dos rendijas. Polarización TM. Revista Mexicana de Física, 40(6), 846-856.

Mata-Méndez, O., Chávez-Rivas, F., \& Ortiz-Acebedo, A. (2008). Diffraction of hermite-gaussian beams by Ronchi and aperiodic rulings. Revista Mexicana de Física, 54(1), 35-41.

Álvarez-Cabanillas. M. A. (1995, December). Tesis Doctoral. "Tratamiento riguroso para la difracción de un haz Hermite-Gauss a través de N-rendijas". ESFM-IPN.

\section{Copyrights}

Copyright for this article is retained by the author(s), with first publication rights granted to the journal.

This is an open-access article distributed under the terms and conditions of the Creative Commons Attribution license (http://creativecommons.org/licenses/by/4.0/). 